JOURNAL OF

SYMPLECTIC GEOMETRY

Volume 8, Number 4, 381-401, 2010

\title{
POSITIVITY OF EQUIVARIANT SCHUBERT CLASSES THROUGH MOMENT MAP DEGENERATION
}

\author{
Catalin Zara
}

For a flag manifold $M=G / B$ with the canonical torus action, the $T$-equivariant cohomology is generated by equivariant Schubert classes, with one class $\tau_{u}$ for every element $u$ of the Weyl group $W$, and these classes are determined by their restrictions to the fixed point set $M^{T} \simeq W$. The main result of this article is a positive formula for computing $\tau_{u}(v)$ in types $A, B$, and $C$. We identify $G / B$ with a generic coadjoint orbit and use a result of Goldin and Tolman to compute $\tau_{u}(v)$ in terms of the induced moment map. Our positive formula, given as a sum indexed by certain saturated chains, follows from a systematic degeneration of the moment map. In type $A$ our formula is equivalent to a classical positive formula that uses summation over certain subwords, but in type $\mathrm{C}$, the two formulas are different.

\section{Nomenclature}

$G$

$B$

$M=G / B$

$T^{\mathbb{C}}, T$

$H_{T}^{*}(M)=H_{T}^{*}(M ; \mathbb{Q})$

$\mathfrak{t}, \mathfrak{t}^{*}$

$\mathcal{B}=\left\{\alpha_{1}, \ldots, \alpha_{n}\right\}$

$0 \prec \beta$

$\omega_{1}, \ldots, \omega_{n}$ connected, complex, semisimple Lie group

Borel subgroup of $G$

flag manifold

maximal complex torus in $B$ and its compact real form

rational $T$-equivariant cohomology of $M$

Lie algebra of $T$ and its dual

simple, positive roots corresponding to $B$

the vector $\beta \in \mathfrak{t}^{*}$ has non-negative coordi-

nates in $\mathcal{B}$

fundamental weights corresponding to

$\alpha_{1}, \ldots, \alpha_{n}$ 


$$
\begin{aligned}
& h(\beta) \\
& =\min \left\{i \mid\left(\omega_{i}, \beta\right) \neq 0\right\}, \text { for } \beta \in \mathfrak{t}^{*} \\
& \eta \in \mathfrak{t}^{*} \\
& \mu_{1}, \ldots, \mu_{n} \\
& \mathcal{O}_{\eta}=G \cdot \eta \\
& \phi_{\eta}: M \simeq \mathcal{O}_{\eta} \rightarrow \mathfrak{t}^{*} \\
& \text { W } \\
& S_{n} \\
& s_{\beta}: \mathfrak{t}^{*} \rightarrow \mathfrak{t}^{*} \\
& s_{i}=s_{\alpha_{i}} \\
& u \beta \\
& h(p, q) \\
& * s_{\beta}, s_{\beta} * \\
& I=\left[i_{1}, \ldots, i_{m}\right] \\
& J=\left[\epsilon_{1} i_{1}, \ldots, \epsilon_{m} i_{m}\right] \\
& s_{I}=s_{i_{1}} \cdots s_{i_{m}} \\
& \ell(w) \\
& u \prec v \\
& I(v)=\left[I_{1}, \ldots, I_{n-1}\right] \\
& \Gamma=(V, E) \\
& e=(u, v) \\
& \alpha: E \rightarrow \mathfrak{t}^{*}, \alpha_{e}=\alpha(u, v) \\
& \mathbb{S}=\mathbb{Q}\left[\alpha_{1}, \ldots, \alpha_{n}\right] \\
& \tau_{u}: W \rightarrow \mathbb{S} \\
& H_{\alpha}^{*}(\Gamma) \\
& \Lambda_{v}^{-} \\
& S C(J, I) \\
& \mathcal{S}(u, I) \supseteq \mathcal{R}(u, I) \\
& \mathcal{A}(u, v) \supseteq \Sigma(u, v) \supseteq \mathcal{C}_{0}(u, v) \\
& E_{\eta}(\gamma) \\
& E_{\mu}(\gamma) \\
& E(\gamma) \\
& F_{I}: \mathcal{A}(u, v) \rightarrow \mathcal{S}(u, I) \\
& \text { generic element in the positive Weyl } \\
& \text { chamber } \\
& \text { coordinates of } \eta \text { in the basis }\left\{\omega_{1}, \ldots, \omega_{n}\right\} \\
& \text { coadjoint orbit through } \eta \\
& \text { moment map for Hamiltonian } T \text {-space } \\
& M \simeq \mathcal{O}_{\eta} \\
& \text { Weyl group of } G \\
& \text { group of permutations of }\{1, \ldots, n\} \\
& \text { reflection generated by the root } \beta \\
& \text { reflection generated by the simple root } \alpha_{i} \\
& \text { action of Weyl group element } u \text { on } \\
& \beta \in \mathfrak{t}^{*} \\
& =\min \left\{i \mid p \omega_{i} \neq q \omega_{i}\right\} \text { for } p, q \in W \\
& \text { Weyl group multiplication by } s_{\beta} \text { to the } \\
& \text { right/left } \\
& \text { word with letters from }\{0,1, \ldots, n\} \\
& \text { subword of } I=\left[i_{1}, \ldots, i_{m}\right] \text {, with } \epsilon_{i} \in\{0,1\} \\
& \text { Weyl group element corresponding to } \\
& I=\left[i_{1}, \ldots, i_{m}\right] \\
& \text { length of Weyl group element } W \\
& u \text { precedes } v \text { in the strong Bruhat order on } \\
& \text { W } \\
& \text { special reduced word for } v \text { (in type } A \text { ) } \\
& \text { graph with vertices } V \text { and oriented edges } E \\
& \text { oriented edge with vertices } u \text { and } v \\
& \text { axial function and its value on the edge } \\
& e=(u, v) \\
& \text { polynomial ring } \\
& \text { (combinatorial) Schubert class } \\
& \text { cohomology ring of }(\Gamma, \alpha) \\
& \text { product of positive roots sent by } v^{-1} \text { into } \\
& \text { negative roots } \\
& \text { contribution of the subword } J \text { of } I \\
& \text { subwords of } I \text { that generate words/reduced } \\
& \text { words for } u \\
& \text { ascending, saturated, and special chains in } \\
& \Gamma \text { from } u \text { to } v \\
& \text { contribution of saturated chain } \gamma \\
& E_{\eta}(\gamma) \text { in terms of } \mu_{1}, \ldots, \mu_{n} \\
& \text { contribution of chain } \gamma \text { after degeneration } \\
& \text { the "delete letters" function }
\end{aligned}
$$




\section{Introduction}

Let $G$ be a connected, complex, semisimple Lie group, $B \subset G$ a Borel subgroup, corresponding to a set of simple roots $\left\{\alpha_{1}, \ldots, \alpha_{n}\right\}$. Let $T^{\mathbb{C}} \subset B$ be a maximal torus, and $T \subset T^{\mathbb{C}}$ a compact real form of $T^{\mathbb{C}}$. The torus $T$ acts on the flag manifold $M=G / B$ by left multiplication on $G$, and the fixed point set $M^{T}$ corresponds bijectively with the Weyl group $W$.

The equivariant cohomology ring $H_{T}^{*}(M)=H_{T}^{*}(M, \mathbb{Q})$ is a free module over $H_{T}^{*}(p t, \mathbb{Q}) \simeq \mathbb{Q}\left[\alpha_{1}, \ldots, \alpha_{n}\right]$, and that is also true for integral, and not just rational coefficients. A basis of this module is given by equivariant Schubert classes, $\left\{\tau_{u}\right\}_{u \in W}$, with a class $\tau_{u}$ for each fixed point $u \in W=M^{T}$. The equivariant Schubert class $\tau_{u} \in H_{T}^{*}(M)$ is the class induced by the $T$-equivariant cycle $X_{u}=\overline{B_{-} u B} / B$, where $B_{-}$is the opposite Borel subgroup. The pull-back map $H_{T}^{*}(M) \rightarrow H_{T}^{*}\left(M^{T}\right)$ is injective, so an equivariant class is determined by its values on $M^{T}$. In particular, the equivariant Schubert classes can be thought of as functions from $W$ to $\mathbb{Q}\left[\alpha_{1}, \ldots, \alpha_{n}\right]$ satisfying certain compatibility conditions (see $[\mathbf{K K}],[\mathbf{G H Z}]$ ).

The integral positivity property ${ }^{1}$ states that $\tau_{u}(v) \in \mathbb{Z}_{\geqslant 0}\left[\alpha_{1}, \ldots, \alpha_{n}\right]$ for all $u, v \in W$; in other words, $\tau_{u}(v)$ is a polynomial in the simple roots $\alpha_{1}, \ldots, \alpha_{n}$, with non-negative integer coefficients. A standard principle is that positive integers count, and effective formulas for quantities that are positive integers or polynomials with such coefficients should be positive and integral: sums of positive integers or polynomials with such coefficients. Such a formula for $\tau_{u}(v)$ appeared in [AJS, Appendix D] (see also [B]), giving $\tau_{u}(v)$ as a sum, indexed by certain reduced subwords, of products of positive roots, hence polynomials in $\alpha_{1}, \ldots, \alpha_{n}$ with non-negative integer coefficients. We will refer to this result as the subword formula.

The main result of this article, formulated in Theorem 6.1 , is a new positive formula for computing $\tau_{u}(v)$ in types $A, B$, and $C$. To obtain this formula we identify $G / B$ with a generic coadjoint orbit and use a recent result of Goldin and Tolman $[\mathbf{G T}]$ to compute $\tau_{u}(v)$ in terms of the induced moment map. Our formula, given as a sum of contributions of certain saturated chains from $u$ to $v$, follows from a systematic degeneration of the moment map, corresponding to degenerating the coadjoint orbit. The resulting formula, which we will refer to as the chain formula, is integral in types $A$ and $C$, but only rational in type $B$ (and for $G_{2}$ ). In type $A$ our formula is equivalent to the subword formula, but in type $C$ the two formulas, while giving the same answer, are different.

The main methods in this paper involve the combinatorics of Weyl groups. The type $A$ formula has been circulated earlier, and motivated a separate project by Sabatini and Tolman $[\mathbf{S T}]$. Their geometric results allowed them

\footnotetext{
${ }^{1} \mathrm{An}$ analogue is valid for the equivariant class corresponding to any $T$-invariant subvariety of $G / B$, as follows from [KM, Theorem D].
} 
to obtain independently, as applications, the same formulas for types $B$ and $C$, but from a different perspective.

The paper is organized as follows. In Section 2 we give a combinatorial description of $\tau_{u}$ and in Section 3 we recall the subword formula. In Section 4 we compute $\tau_{u}(v)$ by applying the Goldin-Tolman result to coadjoint orbits and in Section 5 we use moment map/orbit degeneration to get a simpler formula, in which only some chains have non-zero contributions. In Section 6 we prove that the new formula is positive, integral in types $A$ and $C$ and rational in type $B$, and in Section 7 we give an explicit version in type $A$. We prove the equivalence (for type $A$ ) of the chain formula with the subword formula in Section 9, after constructing, in Section 8, a general map connecting chains and subwords.

\section{Combinatorial Schubert classes}

The injective morphism $H_{T}^{*}(M) \rightarrow H_{T}^{*}\left(M^{T}\right)$ identifies $H_{T}^{*}(M)$ with a subring of $H_{T}^{*}\left(M^{T}\right)=\operatorname{Maps}(W, \mathbb{S})$, where $\mathbb{S}=\mathbb{Q}\left[\alpha_{1}, \ldots, \alpha_{n}\right]$. Not all maps represent classes in $H_{T}^{*}(M)$, and the ones that can be described using a discrete structure involving a regular graph $\Gamma=(V, E)$, and a labeling of the oriented edge of $\Gamma$ by elements of $t^{*}$, the dual of the Lie algebra of $T$.

The vertices $V$ of $\Gamma$ correspond to the Weyl group $W$. Two vertices $u$ and $v$ are joined by an edge if and only if they differ by a reflection $s_{\beta}$, for some positive root $\beta$. Note that $u s_{\beta}=s_{u \beta} u$, so if $u$ and $v$ differ by a reflection on the left, they also differ by a reflection on the right. The notation $u \beta$ means the action of the Weyl group element $u$ on the root $\beta$, and $u s_{\beta}$ is the multiplication in the Weyl group. If $v=u s_{\beta}=s_{u \beta} u$, with $\beta \succ 0$ a positive root, then the oriented edge $e=(u, v)$ of $\Gamma$ is labeled by $\alpha(u, v)=u \beta$ and is called ascending if $\alpha(u, v) \succ 0$. We will also use $\alpha(e), \alpha_{e}$, or $\alpha_{u, v}$ for $\alpha(u, v)$. The pair $(\Gamma, \alpha)$ is called the GKM graph of $(M, T)$.

An assignment $f: W \rightarrow \mathbb{S}=\mathbb{Q}\left[\alpha_{1}, \ldots, \alpha_{n}\right]$ is a cohomology class if

$$
f(v)-f(u) \in \alpha(e) \mathbb{S},
$$

for every edge $e=[u, v]$ of $\Gamma$. The class $f$ is called homogeneous of degree $k$ if, for every $u \in W$, the polynomial $f(u)$ is homogeneous of degree $k$. The cohomology ring $H_{\alpha}^{*}(\Gamma)$ is the graded subring of $\operatorname{Maps}(W, \mathbb{S})$ consisting of all classes. Then

$$
H_{T}^{2 k}(M) \simeq H_{\alpha}^{k}(\Gamma),
$$

and since $H_{T}^{\text {odd }}(M)=0$, that means that $H_{T}^{2 *}(M) \simeq H_{\alpha}^{*}(\Gamma)$. We will in general identify a class $f \in H_{T}^{*}(M)$ with its image in $H_{\alpha}^{*}(\Gamma)$. In particular, we will denote by $\tau_{u}$ both the equivariant Schubert class in $H_{T}^{*}(M)$ and its image in $H_{\alpha}^{*}(\Gamma)$. Sometimes we will refer to $\tau_{u} \in H_{\alpha}^{*}(\Gamma)$ as the combinatorial Schubert class. 
To give the combinatorial description of the (combinatorial) Schubert class $\tau_{u} \in H_{\alpha}^{*}(\Gamma)$, we start by recalling some results concerning the combinatorics of the Weyl group $W$. Most results are valid for general Coxeter groups, and details can be found, for example, in [BB].

Let $R$ be a root system of rank $n$, let $W$ be its Weyl group, and let $\mathcal{B}=\left\{\alpha_{1}, \ldots, \alpha_{n}\right\}$ be a choice of simple roots. Then $\mathcal{B}$ is a basis of $\mathfrak{t}^{*}$, the dual of the Lie algebra of $T$. For a non-zero vector $\beta \in \mathfrak{t}^{*}$ we say that $\beta \succ 0$ if $\beta$ is in the non-negative cone over $\left\{\alpha_{1}, \ldots, \alpha_{n}\right\}$, in other words, if the coordinates of $\beta$ in the basis $\mathcal{B}$ are non-negative.

For $i=1, \ldots, n$, let $s_{i}=s_{\alpha_{i}} \in W$ be the reflection generated $\alpha_{i}$. A word of length $m$ is an array $I=\left[i_{1}, i_{2}, \ldots, i_{m}\right]$ with entries (letters) from $\{0,1, \ldots, n\}$. To each non-empty word $I$ we associate the Weyl group element $s_{I}=s_{i_{1}} s_{i_{2}} \ldots s_{i_{m}}$. (If the word $I$ is empty, then $s_{I}$ is the identity.) A subword of a word $I$ is a word $J=\left[\epsilon_{1} i_{1}, \epsilon_{2} i_{2}, \ldots, \epsilon_{m} i_{m}\right]$, with $\epsilon_{k} \in\{0,1\}$ for all $k=1, \ldots, m$. A word $I$ is reduced if $s_{J} \neq s_{I}$ for all subwords $J$ of $I$ other than $I$ itself. If $v \in W$, then $v=s_{i_{1}} s_{i_{2}} \ldots s_{i_{m}}$ is called a decomposition for $v$, and $I=\left[i_{1}, i_{2}, \ldots, i_{m}\right]$ is a word for $v$. The decomposition is reduced if the word is reduced. All reduced words for $v \in W$ have the same number of letters, and this common number, denoted by $\ell(v)$, is the length of $v$.

We define a partial order on $W$ as follows. If $v=u s_{\beta}$, with $\beta \succ 0$, we define $u \prec v$ if $u \beta \succ 0$. The (strong) Bruhat order on $W$ is the transitive closure of $\prec$. In other words, $u \preccurlyeq v$ if and only if there exists an ascending chain

$$
u=u_{0} \stackrel{* s_{\beta_{1}}}{\longrightarrow} u_{1} \stackrel{* s_{\beta_{2}}}{\longrightarrow} u_{2} \longrightarrow \cdots \stackrel{* s_{\beta_{m}}}{\longrightarrow} u_{m}=v,
$$

where $p \stackrel{* s_{\beta}}{\longrightarrow} q$ means $q=p s_{\beta}$. An equivalent definition can be given in terms of words and subwords: $u \preccurlyeq v$ if and only if every reduced word $I$ for $v$ has a subword that is a word for $u$. Let $\mathcal{F}_{u}=\{v \mid u \preccurlyeq v\}$ be the flow-up from $u$ under the strong Bruhat order.

For $v \in W$, we define

$$
\Lambda_{v}^{-}=\prod\left\{\beta \mid \beta \succ 0 \text { and } v^{-1} \beta \prec 0\right\} .
$$

If $I=\left[i_{1}, \ldots, i_{m}\right]$ is a reduced word for $v$, then the positive roots that are sent by $v^{-1}$ into negative roots are

$$
\alpha_{i_{1}}, s_{i_{1}} \alpha_{i_{2}}, s_{i_{1}} s_{i_{2}} \alpha_{i_{3}}, \ldots, s_{i_{1}} \cdots s_{i_{m-1}} \alpha_{i_{m}},
$$

hence

$$
\Lambda_{v}^{-}=\prod_{j=1}^{m} s_{i_{1}} s_{i_{2}} \cdots s_{i_{j-1}} \alpha_{i_{j}} .
$$

Example 2.1. We illustrate the results above for $G=S L_{n}(\mathbb{C})$, the special linear group of complex matrices with determinant equal to one, with the Borel subgroup $B$ being the subgroup of upper triangular matrices. Then 
$M=G / B$ is the manifold of complete flags in $\mathbb{C}^{n}$. The fixed points of the $T$-action correspond bijectively to permutation matrices, hence $M^{T}$ is identified with $W=S_{n}$, the set of permutations of $\{1, \ldots, n\}$. We use the one-line notation $u=u(1) u(2) \ldots u(n)$ for permutations. The simple roots are $\alpha_{i}=x_{i}-x_{i+1}$ and the positive roots are $\alpha_{i j}=x_{i}-x_{j}=\alpha_{i}+\cdots+\alpha_{j-1}$, for $1 \leqslant i<j \leqslant n$. The Weyl group $W=S_{n}$ acts on roots by permuting the indices of the $x$ variables. The reflection $s_{\alpha_{i j}}$ corresponds to the transposition $(i, j)$ that swaps $i$ and $j$; if $j=i+1$, we denote $s_{\alpha_{i j}}$ by $s_{i}$ and call it a simple transposition. By convention, $s_{0}$ is the identity of $S_{n}$.

For a permutation $v \in S_{n}$, the set of positive roots that are sent by $v^{-1}$ into negative roots corresponds bijectively to the set of inversions-as-values,

$$
\operatorname{Inv}(v)=\{(v(j), v(i)) \mid i<j, v(i)>v(j)\} .
$$

If $I=\left[i_{1}, \ldots, i_{m}\right]$ is a reduced word for $v$, then

$$
\Lambda_{v}^{-}=\prod_{(a, b) \in \operatorname{Inv}(v)}\left(x_{a}-x_{b}\right)=\prod_{k=1}^{m} s_{i_{1}} \cdots s_{i_{k-1}} \alpha_{i_{k}} .
$$

If $v=u(i, j)$ with $i<j$, then $u \prec v$ in the strong Bruhat order if and only if $u(i)<u(j)$. In other words, $u \prec v=u(i, j)$ if $(u(i), u(j)) \notin \operatorname{Inv}(u)$, or, equivalently, if $(v(i), v(j)) \in \operatorname{Inv}(v)$.

The description of the combinatorial Schubert class $\tau_{u}$ is the following.

Proposition 2.1. For every $u \in W$, the class $\tau_{u} \in H_{\alpha}^{*}(\Gamma)$ is the unique class satisfying the following properties:

(1) $\tau_{u}$ is homogeneous, of degree $\ell(u)$, the length of $u$;

(2) $\tau_{u}$ is supported on $\mathcal{F}(u)$, the flow-up from $u$;

(3) $\tau_{u}$ is normalized by $\tau_{u}(u)=\Lambda_{u}^{-}$.

\section{Subword formula}

In this section we recall the subword integral positive formula. Let $u, v \in W$, with $u \preccurlyeq v$, and let $I=\left[i_{1}, i_{2}, \ldots, i_{m}\right]$ be a reduced word for $v$. For a subword $J=\left[\epsilon_{1} i_{1}, \epsilon_{2} i_{2}, \ldots, \epsilon_{m} i_{m}\right]$ of $I$, define the subword contribution $\mathrm{SC}(J, I)$ as the following product of positive roots:

$$
\begin{aligned}
\operatorname{SC}(J, I) & =\prod_{j=1}^{m}\left[s_{i_{1}} s_{i_{2}} \cdots s_{i_{j-1}} \alpha_{i_{j}}\right]^{\epsilon_{j}}=\prod_{\substack{j=1 \\
\epsilon_{j}=1}}^{m} s_{i_{1}} s_{i_{2}} \cdots s_{i_{j-1}} \alpha_{i_{j}} \\
& =\Lambda_{v}^{-} \cdot \prod_{\substack{j=1 \\
\epsilon_{j}=0}}^{m} \frac{1}{s_{i_{1}} s_{i_{2}} \cdots s_{i_{j-1}} \alpha_{i_{j}}}
\end{aligned}
$$


hence $\mathrm{SC}(J, I)$ is obtained from $\Lambda_{v}^{-}$by canceling the positive roots generated by the deleted letters $\left(\epsilon_{j}=0\right)$. Let $\mathcal{R}(u, I)$ be the set of subwords of $I$ that, after deleting the zeroes, become reduced words for $u$.

Theorem 3.1 ([AJS, B]). Let $u, v \in W$ and let $I=\left[i_{1}, \ldots, i_{m}\right]$ be a reduced word for $v$. The value at $v$ of the Schubert class $\tau_{u}$ is given by

$$
\tau_{u}(v)=\sum_{J \in \mathcal{R}(u, I)} \operatorname{SC}(J, I)=\sum_{J \in \mathcal{R}(u, I)} \prod_{\substack{j=1 \\ \epsilon_{j}=1}}^{m} s_{i_{1}} s_{i_{2}} \cdots s_{i_{j-1}} \alpha_{i_{j}} .
$$

Example 3.1. Let $u=2143$ and $v=3421$ in $S_{4}$. A reduced word for $v$ is $I=[2,1,3,2,3]$. There are two subwords of $I$ that are reduced words for $u$ after deleting all the zeroes: $J_{1}=[0,1,3,0,0]$ and $J_{2}=[0,1,0,0,3]$. Their contributions are

$$
\begin{aligned}
\mathrm{SC}\left(J_{1}, I\right) & =\left[\alpha_{2}\right]^{0} \cdot\left[s_{2} \alpha_{1}\right]^{1} \cdot\left[s_{2} s_{1} \alpha_{3}\right]^{1} \cdot\left[s_{2} s_{1} s_{3} \alpha_{2}\right]^{0} \cdot\left[s_{2} s_{1} s_{3} s_{2} \alpha_{3}\right]^{0} \\
& =\left[s_{2} \cdot\left(x_{1}-x_{2}\right)\right] \cdot\left[s_{2} s_{1} \cdot\left(x_{3}-x_{4}\right)\right]=\left(x_{1}-x_{3}\right)\left(x_{2}-x_{4}\right) \\
& =\left(\alpha_{1}+\alpha_{2}\right)\left(\alpha_{2}+\alpha_{3}\right) \\
\operatorname{SC}\left(J_{2}, I\right) & =\left[\alpha_{2}\right]^{0} \cdot\left[s_{2} \alpha_{1}\right]^{1} \cdot\left[s_{2} s_{1} \alpha_{3}\right]^{0} \cdot\left[s_{2} s_{1} s_{3} \alpha_{2}\right]^{0} \cdot\left[s_{2} s_{1} s_{3} s_{2} \alpha_{3}\right]^{1} \\
& =\left[s_{2} \cdot\left(x_{1}-x_{2}\right)\right] \cdot\left[s_{2} s_{1} s_{3} s_{2} \cdot\left(x_{3}-x_{4}\right)\right]=\left(x_{1}-x_{3}\right)\left(x_{1}-x_{2}\right) \\
& =\left(\alpha_{1}+\alpha_{2}\right) \alpha_{1} .
\end{aligned}
$$

Therefore

$$
\tau_{2143}(3421)=\operatorname{SC}\left(J_{1}, I\right)+\operatorname{SC}\left(J_{2}, I\right)=\left(\alpha_{1}+\alpha_{2}\right)\left(\alpha_{1}+\alpha_{2}+\alpha_{3}\right) .
$$

\section{The Goldin-Tolman formula}

Goldin and Tolman $[\mathbf{G T}]$ recently proved a formula for computing the values of what they call canonical classes, valid for more general spaces. ${ }^{2}$ Their classes satisfy conditions 1 and 3 in Proposition 2.1, but condition 2 is replaced by $\tau_{u}(v)=0$ if $\ell(v) \leqslant \ell(u)$ and $v \neq u$. Since the length function is strictly increasing with respect to the Bruhat order $(u \prec v \rightarrow \ell(u)<\ell(v))$, it turns out that, in the case of flag manifolds, the canonical generators are the equivariant Schubert classes and the Goldin-Tolman formula can be used to compute the values $\tau_{u}(v)$ for $u, v \in W$.

The Goldin-Tolman formula involves two more ingredients. The first ingredient is the subgraph $\Gamma_{0}$ of $\Gamma$, having the same vertices as $\Gamma$, but only the edges $e=(u, v)$ of $\Gamma$ for which $\ell(v)=\ell(u) \pm 1$. If $u$ and $v$ are elements in $W$, let $\Sigma(u, v)$ be the set of ascending chains in $\Gamma_{0}$ from $u$ to $v$. These are the maximal length ascending chains in $\Gamma$ from $u$ to $v$, and we call such chains saturated, because they are the saturated chains from $u$ to $v$ in the

\footnotetext{
${ }^{2}$ The flag manifold case of this formula also appeared, in implicit form, in $[\mathbf{K}]$.
} 
poset $(W, \prec)$. Every edge of such a chain will be considered oriented, with the orientation that makes it an ascending edge.

The second ingredient is a moment map. The Lie algebra $\mathfrak{g}$ of $G$ can be canonically identified with its dual $\mathfrak{g}^{*}$ using the Killing form, and that allows us to regard $\mathfrak{t}^{*}$ as a subspace of $\mathfrak{g}^{*}$. Let $\eta \in \mathfrak{t}^{*} \subset \mathfrak{g}^{*}$ be in the interior of the positive Weyl chamber, and let $\mathcal{O}_{\eta}=G \cdot \eta \subset \mathfrak{g}^{*}$ be the coadjoint orbit through $\eta$. The stabilizer of $\eta$ is $B$, and hence $\mathcal{O}_{\eta} \simeq G / B=M$ as $T$-spaces.

The coadjoint orbit $\mathcal{O}_{\eta}$ is a Hamiltonian $G$-space, with moment map given by the inclusion $\mathcal{O}_{\eta} \hookrightarrow \mathfrak{g}^{*}$. Therefore it is also a Hamiltonian $T$-space, with moment map given by inclusion followed by projection onto $\mathfrak{t}^{*}$. With the symplectic structure induced by the identification $G / B \simeq \mathcal{O}_{\eta}$, the flag manifold $M=G / B$ is a Hamiltonian $T$-space, with moment map $\phi_{\eta}: G / B \rightarrow \mathfrak{t}^{*}$. If $P_{w}$ is the fixed point corresponding to the element $w \in W$ of the Weyl group, then $\phi_{\eta}\left(P_{w}\right)=w \cdot \eta$. Identifying the fixed point $P_{w}$ with the Weyl group element $w$, we get a map $\phi_{\eta}: W \rightarrow \mathfrak{t}^{*}$, given by $\phi_{\eta}(w)=w \eta$.

Applying the Goldin-Tolman formula we get the following result.

Theorem 4.1. If $\eta \in \mathfrak{t}^{*}$ is in the positive Weyl chamber, then

$$
\tau_{u}(v)=\sum_{\gamma \in \Sigma(u, v)} E_{\eta}(\gamma)
$$

where, for every saturated chain,

$$
\gamma: u=u_{0} \longrightarrow u_{1} \longrightarrow \cdots \longrightarrow u_{m}=v
$$

in $\Sigma(u, v)$, the contribution $E_{\eta}(\gamma)$ is given by

$$
E_{\eta}(\gamma)=\Lambda_{v}^{-} \prod_{k=1}^{m}\left(\frac{1}{\alpha\left(u_{k-1}, u_{k}\right)} \cdot \frac{\phi_{\eta}\left(u_{k}\right)-\phi_{\eta}\left(u_{k-1}\right)}{\phi_{\eta}(v)-\phi_{\eta}\left(u_{k-1}\right)}\right) .
$$

There are two important features in formula (4.1). The first is that each term $E_{\eta}(\gamma)$ depends on $\eta$, but the sum doesn't. The second is that each term is a rational expression, given by (4.3), but the sum is a polynomial. Therefore cancelation must occur when summing, and a first indication of how that happens is the following.

Lemma 4.1. If $p, q \in W$ and $q=p s_{\beta}$ with $\beta \succ 0$, then

$$
\frac{\phi_{\eta}(q)-\phi_{\eta}(p)}{\alpha(p, q)}<0 \text {. }
$$

Proof.

$$
\frac{\phi_{\eta}(q)-\phi_{\eta}(p)}{\alpha(p, q)}=\frac{p s_{\beta} \eta-p \eta}{p \beta}=-\langle\eta, \beta\rangle=-\frac{2(\eta, \beta)}{(\beta, \beta)}<0,
$$

since $\beta$ is a positive root and $\eta$ is in the positive Weyl chamber. 
Hence, if $\gamma$ is the chain (4.2) and $u_{k}=u_{k-1} s_{\beta_{k}}$ with $\beta_{k} \succ 0$, then

$$
E_{\eta}(\gamma)=\Lambda_{v}^{-} \prod_{k=1}^{m} \frac{\left\langle\eta, \beta_{k}\right\rangle}{u_{k-1} \eta-v \eta}
$$

Lemma 4.2. Let $p \prec q$, let $\eta$ be in the closure of the positive Weyl chamber and let $\beta=p \eta-q \eta$. Then $\beta \succcurlyeq 0$. If $\beta \succ 0$, then $p^{-1} \beta \succ 0$ and $q^{-1} \beta \prec 0$.

Proof. If $p \prec q$, then there exists an ascending chain

$$
p=p_{0} \stackrel{* s_{\beta_{1}}}{\longrightarrow} p_{1} \stackrel{* s_{\beta_{2}}}{\longrightarrow} p_{2} \rightarrow \cdots \stackrel{* s_{\beta_{m}}}{\longrightarrow} p_{m}=q
$$

with $\beta_{k} \succ 0$ and $p_{k-1} \beta_{k} \succ 0$. Then

$$
\begin{aligned}
p \eta-q \eta & =\left(p \eta-p_{1} \eta\right)+\cdots+\left(p_{k-1} \eta-p_{k} \eta\right)+\cdots+\left(p_{m-1} \eta-q \eta\right) \\
& =\left\langle\eta, \beta_{1}\right\rangle p_{0} \beta_{1}+\cdots+\left\langle\eta, \beta_{k}\right\rangle p_{k-1} \beta_{k}+\cdots+\left\langle\eta, \beta_{m}\right\rangle p_{m-1} \beta_{m}
\end{aligned}
$$

and each of the terms in the last sum is in the non-negative cone generated by the simple roots, hence $\beta \succcurlyeq 0$. Moreover, $\beta=0$ if and only if all the factors $\left\langle\eta, \beta_{k}\right\rangle$ are zero.

We have $p^{-1} \beta=\eta-p^{-1} q \eta=i d \eta-p^{-1} q \eta$, where $i d$ is the identity in the Weyl group. Since $i d \prec p^{-1} q$, if $\beta \succ 0$ then $p^{-1} \beta \succ 0$, and similarly, $q^{-1} \eta=-\left(i d \eta-q^{-1} p \eta\right) \prec 0$.

Therefore $E_{\eta}(\gamma)$ is a ratio of two polynomials in the simple roots $\alpha_{1}, \ldots, \alpha_{n}$, homogeneous and with non-negative coefficients. Moreover, if $u_{k-1} \eta-v \eta$ is a multiple of a root, then it cancels (over $\mathbb{R}_{>0}$ ) one of the factors of $\Lambda_{v}^{-}$.

Let $\left\{\omega_{1}, \ldots, \omega_{n}\right\}$ be the fundamental weights corresponding to the simple roots $\left\{\alpha_{1}, \ldots, \alpha_{n}\right\}$, defined by the conditions

$$
\left\langle\omega_{i}, \alpha_{j}\right\rangle=\delta_{i j} \text {, or equivalently }, \quad\left(\omega_{i}, \alpha_{j}\right)=\frac{1}{2}\left(\alpha_{i}, \alpha_{i}\right) \delta_{i j} .
$$

Then $\left\{\omega_{1}, \ldots, \omega_{n}\right\}$ is a basis of $\mathfrak{t}^{*}$, and $\eta=\mu_{1} \omega_{1}+\cdots+\mu_{n} \omega_{n} \in \mathfrak{t}^{*}$ is in the positive Weyl chamber if and only if $\mu_{j}>0$ for all $j=1, \ldots, n$.

Then the contribution $E_{\mu}(\gamma)=E_{\mu_{1} \omega_{1}+\cdots+\mu_{n} \omega_{n}}(\gamma)$ of the chain $\gamma$ is

$$
E_{\mu}(\gamma)=\Lambda_{v}^{-} \prod_{k=1}^{m} \frac{\sum_{i=1}^{n}\left\langle\omega_{i}, \beta_{k}\right\rangle \mu_{i}}{\sum_{i=1}^{n}\left(\sum_{j=k}^{m}\left\langle\omega_{i}, \beta_{j}\right\rangle u_{j-1} \beta_{j}\right) \mu_{i}} .
$$

Example 4.1. Let $M=\mathrm{SL}_{3}(\mathbb{C}) / B$ be the manifold of complete flags in $\mathbb{C}^{3}$, let $u=s_{1}=213$ and $v=s_{1} s_{2} s_{1}=321$. Then $\ell(321)=3$, and

$$
\Lambda_{v}^{-}=\alpha_{1} \alpha_{2}\left(\alpha_{1}+\alpha_{2}\right) \text {. }
$$

There are two saturated chains from $u$ to $v$,

$$
\begin{array}{ll}
\gamma_{1}: & s_{1} \stackrel{* s_{\alpha_{1}+\alpha_{2}}}{\longrightarrow} s_{2} s_{1} \stackrel{* s_{\alpha_{2}}}{\longrightarrow} s_{1} s_{2} s_{1}, \\
\gamma_{2}: & s_{1} \stackrel{* s_{\alpha_{2}}}{\longrightarrow} s_{1} s_{2} \stackrel{* s_{\alpha_{1}}}{\longrightarrow} s_{1} s_{2} s_{1},
\end{array}
$$


and their contributions are

$$
\begin{aligned}
& E_{\mu}\left(\gamma_{1}\right)=\alpha_{1} \alpha_{2}\left(\alpha_{1}+\alpha_{2}\right) \cdot \frac{\mu_{1}+\mu_{2}}{\alpha_{2} \mu_{1}+\left(\alpha_{1}+\alpha_{2}\right) \mu_{2}} \cdot \frac{\mu_{2}}{\alpha_{1} \mu_{2}}=\frac{\alpha_{2}\left(\alpha_{1}+\alpha_{2}\right)\left(\mu_{1}+\mu_{2}\right)}{\alpha_{2} \mu_{1}+\left(\alpha_{1}+\alpha_{2}\right) \mu_{2}}, \\
& E_{\mu}\left(\gamma_{2}\right)=\alpha_{1} \alpha_{2}\left(\alpha_{1}+\alpha_{2}\right) \cdot \frac{\mu_{2}}{\alpha_{2} \mu_{1}+\left(\alpha_{1}+\alpha_{2}\right) \mu_{2}} \cdot \frac{\mu_{1}}{\alpha_{2} \mu_{1}}=\frac{\alpha_{1}\left(\alpha_{1}+\alpha_{2}\right) \mu_{2}}{\alpha_{2} \mu_{1}+\left(\alpha_{1}+\alpha_{2}\right) \mu_{2}} .
\end{aligned}
$$

Then

$$
\tau_{u}(v)=E_{\mu}\left(\gamma_{1}\right)+E_{\mu}\left(\gamma_{2}\right)=\alpha_{1}+\alpha_{2} .
$$

\section{Limits and chain contributions}

Note that, in Example 4.1, both $E_{\mu}\left(\gamma_{1}\right)$ and $E_{\mu}\left(\gamma_{2}\right)$ are rational expressions in $\alpha$ 's and $\mu$ 's, but their sum is a polynomial expression in the $\alpha$ variables only. In this section we show that we can eliminate the $\mu$ variables in (4.5) by sending them to 0 , one component at a time. ${ }^{3}$

The contribution (4.3) of the chain (4.2) can be written as

$$
E_{\mu}(\gamma)=\Lambda_{v}^{-} \prod_{k=1}^{m} \frac{Q_{\mu}\left(u_{k-1}, u_{k}, v\right)}{\alpha\left(u_{k-1}, u_{k}\right)},
$$

where, for $p \prec q \preccurlyeq r$ in $W$, we define

$$
Q_{\mu}(p, q, r)=\frac{p \eta-q \eta}{p \eta-r \eta}=\frac{\sum_{i=1}^{n}\left(p \omega_{i}-q \omega_{i}\right) \mu_{i}}{\sum_{i=1}^{n}\left(p \omega_{i}-r \omega_{i}\right) \mu_{i}} .
$$

Lemma 5.1. For $p, q \in W$, let

$$
h(p, q)=\min \left\{i \mid p \omega_{i} \neq q \omega_{i}\right\},
$$

with the convention that $h(p, p)=\infty$.

(1) If $p^{-1} q=s_{i_{1}} s_{i_{2}} \cdots s_{i_{k}}$ is a reduced decomposition, then

$$
h(p, q)=\min \left\{i_{1}, \ldots, i_{k}\right\} .
$$

(2) If $p \prec q \prec r$ and $p \omega_{i}=r \omega_{i}$, then $p \omega_{i}=q \omega_{i}=r \omega_{i}$.

(3) If $p \prec q \prec r$, then

$$
h(p, r) \leqslant h(p, q) \quad \text { and } \quad h(q, r) \leqslant h(p, q) .
$$

(4) If $q=p s_{\beta}$ with $\beta \succ 0$ and $p \beta \succ 0$, then $h(p, q)=h(\beta)$, where, for nonzero $\beta=\beta^{1} \alpha_{1}+\cdots+\beta^{n} \alpha_{n}$ in $\mathfrak{t}^{*}$, we define $h(\beta)=\min \left\{i \mid \beta^{i} \neq 0\right\}$.

Proof. Let $j=h(p, q)$ and $\omega_{i}$ a fundamental weight. Then (4.4) becomes

$$
\omega_{i}-p^{-1} q \omega_{i}=\delta_{i_{1}, i} \alpha_{i_{1}}+\delta_{i_{2}, i} s_{i_{1}} \alpha_{i_{2}}+\cdots+\delta_{i_{k}, i} s_{i_{1}} s_{i_{2}} \cdots s_{i_{k-1}} \alpha_{i_{k}} .
$$

By definition, if $i<j$, then $\omega_{i}=p^{-1} q \omega_{i}$, and since all the non-zero terms in (5.1) are positive roots, it follows that $s_{i}$ cannot appear in the reduced decomposition of $p^{-1} q$. If $i=j$, then $\omega_{j} \neq p^{-1} q \omega_{j}$, hence $s_{j}$ must appear

\footnotetext{
${ }^{3}$ The geometric interpretation of this operation is one of the main ideas in $[\mathbf{S T}]$.
} 
in the decomposition of $p^{-1} q$. Therefore, $j=h(p, q)$ is the minimal letter that appears in a reduced word for $p^{-1} q$ and $p^{-1} q$ is in the subgroup of $W$ generated by $s_{j}, s_{j+1}, \ldots, s_{n}$.

For every $i=1, \ldots, n$ we have $p \omega_{i}-r \omega_{i}=\left(p \omega_{i}-q \omega_{i}\right)+\left(q \omega_{i}-r \omega_{i}\right)$, and since $p \prec q \prec r$, both $p \omega_{i}-q \omega_{i}$ and $q \omega_{i}-r \omega_{i}$ are in the non-negative cone generated by the simple roots. Therefore, if $p \omega_{i}-r \omega_{i}=0$, then $p \omega_{i}-q \omega_{i}=0$ and $q \omega_{i}-r \omega_{i}=0$. Then $\left\{i \mid p \omega_{i}=r \omega_{i}\right\} \subset\left\{i \mid p \omega_{i}=q \omega_{i}\right\}$, which implies $h(p, r) \leqslant h(p, q)$. Similarly we get $h(p, r) \leqslant h(q, r)$.

If $q=p s_{\beta}$, then

$$
\left\{i \mid p \omega_{i} \neq p s_{\beta} \omega_{i}\right\}=\left\{i \mid \omega_{i} \neq s_{\beta} \omega_{i}\right\}=\left\{i \mid\left(\omega_{i}, \beta\right) \neq 0\right\}=\left\{i \mid \beta^{i} \neq 0\right\},
$$

hence $h(p, q)=h(\beta)$.

Consequently, if the coefficient of some $\mu_{i}$ in the denominator is zero, then the coefficient of $\mu_{i}$ in the numerator is also zero. This implies that

$$
Q_{\mu}^{0}(p, q, r) \stackrel{\text { def }}{=} \lim _{\mu_{1} \rightarrow 0}\left(\lim _{\mu_{2} \rightarrow 0}\left(\cdots\left(\lim _{\mu_{n} \rightarrow 0} Q_{\mu}(p, q, r)\right) \cdots\right)\right)
$$

is well defined. Moreover,

$$
Q_{\mu}^{0}(p, q, r)=\left\{\begin{aligned}
\frac{p \omega_{i}-q \omega_{i}}{p \omega_{i}-r \omega_{i}}, & \text { if } h(p, r)=h(p, q)=i \\
0, & \text { if } h(p, r)<h(p, q) .
\end{aligned}\right.
$$

In particular, let $p=u_{k-1}, q=u_{k}=u_{k-1} \beta_{k}$, and $r=v$. Then

$$
\frac{Q_{\mu}^{0}\left(u_{k-1}, u_{k}, v\right)}{\alpha\left(u_{k-1}, u_{k}\right)}=\left\{\begin{aligned}
\frac{\left\langle\omega_{i}, \beta_{k}\right\rangle}{u_{k-1} \omega_{i}-v \omega_{i}}, & \text { if } h\left(u_{k-1}, v\right)=h\left(u_{k-1}, u_{k}\right)=i \\
0, & \text { if } h\left(u_{k-1}, v\right)<h\left(u_{k-1}, u_{k}\right) .
\end{aligned}\right.
$$

Therefore,

$$
E(\gamma) \stackrel{\text { def }}{=} \lim _{\mu_{1} \rightarrow 0}\left(\lim _{\mu_{2} \rightarrow 0}\left(\cdots\left(\lim _{\mu_{n} \rightarrow 0} E_{\mu}(\gamma)\right) \cdots\right)\right)
$$

is well-defined, and is zero whenever $h\left(u_{k-1}, v\right)<h\left(u_{k-1}, u_{k}\right)$ for some $k$. If $h\left(u_{k-1}, u_{k}\right)=h\left(u_{k-1}, v\right)=i_{k}$ for all $k=1, \ldots, m$, then

$$
E(\gamma)=\Lambda_{v}^{-} \prod_{k=1}^{m} \frac{\left\langle\omega_{i_{k}}, \beta_{k}\right\rangle}{u_{k-1} \omega_{i_{k}}-v \omega_{i_{k}}} \neq 0
$$

We now take a closer look at the set of chains

$$
\mathcal{C}_{0}(u, v)=\left\{\gamma \in \Sigma(u, v) \mid h\left(u_{k-1}, u_{k}\right)=h\left(u_{k-1}, v\right) \text { for all } k=1, \ldots, m\right\}
$$

with non-zero contribution after taking the limits. These chains have a simple description. 
Lemma 5.2. Let $\gamma \in \Sigma(u, v)$ be the saturated chain

$$
\gamma: u=u_{0} \stackrel{* s_{\beta_{1}}}{\longrightarrow} u_{1} \stackrel{* s_{\beta_{2}}}{\longrightarrow} \cdots \stackrel{* s_{\beta_{m}}}{\longrightarrow} u_{m}=v .
$$

Then $\gamma \in \mathcal{C}_{0}(u, v)$ if and only if $h\left(\beta_{1}\right) \leqslant h\left(\beta_{2}\right) \leqslant \cdots \leqslant h\left(\beta_{m}\right)$.

Proof. If $\gamma \in \mathcal{C}_{0}(u, v)$, then, using Lemma 5.1, we get

$$
h\left(\beta_{k+1}\right)=h\left(u_{k}, u_{k+1}\right)=h\left(u_{k}, v\right) \geqslant h\left(u_{k-1}, v\right)=h\left(u_{k-1}, u_{k}\right)=h\left(\beta_{k}\right)
$$

for all $k=1, \ldots, m-1$, proving one implication.

Conversely, let $\gamma \in \Sigma(u, v)$ such that $h\left(\beta_{1}\right) \leqslant h\left(\beta_{2}\right) \leqslant \cdots \leqslant h\left(\beta_{m}\right)$. Let $k \in\{1, \ldots, m\}$ be fixed. If $i<h\left(\beta_{k}\right)$, then $i<h\left(\beta_{j}\right)=h\left(u_{j-1}, u_{j}\right)$ for all $j \geqslant k$, hence $u_{j-1} \omega_{i}=u_{j} \omega_{i}$ for all $j \geqslant k$. Therefore $u_{k-1} \omega_{i}=u_{m} \omega_{i}=v \omega_{i}$, hence $h\left(u_{k-1}, v\right) \geqslant h\left(\beta_{k}\right)=h\left(u_{k-1}, u_{k}\right)$. But $h\left(u_{k-1}, v\right) \leqslant h\left(u_{k-1}, u_{k}\right)$ by Lemma 5.1, and the double inequality implies that $h\left(u_{k-1}, v\right)=h\left(u_{k-1}, u_{k}\right)$ for all $k$, and therefore $\gamma \in \mathcal{C}_{0}(u, v)$.

Example 5.1. Returning to Example 4.1, we see that

$$
\begin{aligned}
& E\left(\gamma_{1}\right)=\lim _{\mu_{1} \rightarrow 0}\left(\lim _{\mu_{2} \rightarrow 0} \frac{\alpha_{2}\left(\alpha_{1}+\alpha_{2}\right)\left(\mu_{1}+\mu_{2}\right)}{\alpha_{2} \mu_{1}+\left(\alpha_{1}+\alpha_{2}\right) \mu_{2}}\right)=\alpha_{1}+\alpha_{2} \neq 0, \\
& E\left(\gamma_{2}\right)=\lim _{\mu_{1} \rightarrow 0}\left(\lim _{\mu_{2} \rightarrow 0} \frac{\alpha_{1}\left(\alpha_{1}+\alpha_{2}\right) \mu_{2}}{\alpha_{2} \mu_{1}+\left(\alpha_{1}+\alpha_{2}\right) \mu_{2}}\right)=0,
\end{aligned}
$$

as expected, since, for $\gamma_{1}$ we have $h\left(\alpha_{1}+\alpha_{2}\right)=1 \leqslant 2=h\left(\alpha_{2}\right)$ and for $\gamma_{2}$ we have $h\left(\alpha_{2}\right)=2 \nless 1=h\left(\alpha_{1}\right)$.

\section{Chain formula}

Up to this point, there have been no restrictions regarding the order in which the simple roots $\alpha_{1}, \ldots, \alpha_{n}$ are listed. The next results, however, requires that the simple roots be listed in a specific order, as shown in the following Dynkin diagrams:

$$
\begin{array}{ll}
\text { Type } A: & \alpha_{1}-\alpha_{2}-\cdots-\alpha_{n-1}-\alpha_{n}, \\
\text { Type } B: & \alpha_{1}-\alpha_{2}-\cdots-\alpha_{n-1} \rightleftharpoons \alpha_{n}, \\
\text { Type } C: & \alpha_{1}-\alpha_{2}-\cdots-\alpha_{n-1} \longleftarrow \alpha_{n} .
\end{array}
$$

We are now ready to formulate the main result of this paper. Recall that $\mathcal{C}_{0}(u, v)$ is the set of saturated chains

$$
\gamma: u=u_{0} \stackrel{* s_{\beta_{1}}}{\longrightarrow} u_{1} \stackrel{* s_{\beta_{2}}}{\longrightarrow} u_{2} \rightarrow \cdots \rightarrow u_{m-1} \stackrel{* s_{\beta_{m}}}{\longrightarrow} u_{m}=v
$$

that satisfy the condition

$$
h\left(\beta_{1}\right) \leqslant h\left(\beta_{2}\right) \leqslant \cdots \leqslant h\left(\beta_{m}\right),
$$


and for each such a chain $\gamma$, by (5.2),

$$
E(\gamma)=\Lambda_{v}^{-} \prod_{k=1}^{m} \frac{\left\langle\omega_{i_{k}}, \beta_{k}\right\rangle}{u_{k-1} \omega_{i_{k}}-v \omega_{i_{k}}},
$$

where $i_{k}=h\left(\beta_{k}\right)$ for $k=1, \ldots, m$.

Theorem 6.1. If $u, v \in W$ then the restriction of $\tau_{u}$ at $v$ is given by

$$
\tau_{u}(v)=\sum_{\gamma \in \mathcal{C}_{0}(u, v)} E(\gamma)=\sum_{\gamma \in \mathcal{C}_{0}(u, v)} \Lambda_{v}^{-} \prod_{k=1}^{m} \frac{\left\langle\omega_{i_{k}}, \beta_{k}\right\rangle}{u_{k-1} \omega_{i_{k}}-v \omega_{i_{k}}} .
$$

If the simple roots are ordered as in (6.1), then, in types $A$ and $C$

$$
E(\gamma) \in \mathbb{Z}_{\geqslant 0}\left[\alpha_{1}, \ldots, \alpha_{n}\right],
$$

and in type $B$

$$
E(\gamma) \in \frac{1}{2^{m}} \mathbb{Z}_{\geqslant 0}\left[\alpha_{1}, \ldots, \alpha_{n}\right]
$$

Therefore (6.4) is a positive integral formula for computing $\tau_{u}(v)$ in types $A$ and $C$, and a positive, but only rational, formula in type $B$.

Proof. The right-hand side of the Goldin-Tolman formula (4.1) is independent of the particular choice of $\eta$ in the positive chamber (and hence of $\mu$ with strictly positive components). Each term in the right-hand side is welldefined after sending the components of $\mu$ to zero, one at a time in reverse lexicographic order. If $\gamma \notin \mathcal{C}_{0}(u, v)$, then the limit is zero, and if $\gamma \in \mathcal{C}_{0}(u, v)$, then the limit is $E(\gamma)$, and this proves (6.4).

With the ordering (6.1), if $\beta$ is a positive root and $h(\beta)=i$, then $\left\langle\omega_{i}, \beta\right\rangle$ is a positive integer, so the product in the numerator of $(5.2)$ is a positive integer. It remains to show that:

(1) Each factor in the denominator of (5.2) cancels (over $\mathbb{Z}_{>0}$ in types $A$ and $C$, and over $\frac{1}{2} \mathbb{Z}_{>0}$ in type $B$ ) one of the factors in $\Lambda_{v}^{-}$, and

(2) The factors in the denominator of (5.2) are pairwise independent.

These statements are consequences of the following technical lemmas:

Lemma 6.1. If $u \in W$ and $j=h(i d, u)$, then,

In type $A$ : $u$ has a reduced decomposition of the form

$$
u=s_{k} s_{k-1} \cdots s_{j} w
$$

with $j \leqslant k \leqslant n$ and $h(i d, w)>j$.

In type $B$ or $C$ : u has either a reduced decomposition (6.5) or a reduced decomposition of the form

$$
u=s_{t} s_{t+1} \cdots s_{n-1} s_{n} s_{n-1} \cdots s_{j+1} s_{j} w,
$$

with $j \leqslant t<n$ and $h(i d, w)>j$, but not both. 
Proof. Start with a reduced word for $u$. By Lemma 5.1, $j$ is the smallest letter in that word. One can use the Coxeter relations to eliminate all but one (in type $A$ ), or at most two (in types $B$ and $C$ ) occurrences of $j$.

Lemma 6.2. If $u$ has a decomposition of the form (6.5), then $\omega_{j}-u \omega_{j}= \begin{cases}\alpha_{j}+\cdots+\alpha_{k}, & \text { in types } A, C \text { if } k \leqslant n \text { and } B \text { if } k<n, \\ \alpha_{j}+\cdots+\alpha_{n-1}+2 \alpha_{n}, & \text { in type } B \text { for } k=n .\end{cases}$

If $u$ has a decomposition of the form (6.6) with $t>j$, then $\omega_{j}-u \omega_{j}= \begin{cases}\alpha_{j}+\cdots+\alpha_{t-1}+2\left(\alpha_{t}+\cdots+\alpha_{n}\right), & \text { in type } B, \\ \alpha_{j}+\cdots+\alpha_{t-1}+2\left(\alpha_{t}+\cdots+\alpha_{n-1}\right)+\alpha_{n}, & \text { in type } C .\end{cases}$

If $u$ has a decomposition of the form (6.6) with $t=j$, then

$$
\omega_{j}-u \omega_{j}= \begin{cases}2\left(\alpha_{j}+\cdots+\alpha_{n}\right), & \text { in type } B, \\ 2\left(\alpha_{j}+\cdots+\alpha_{n-1}\right)+\alpha_{n}, & \text { in type } C .\end{cases}
$$

Proof. Since $h(i d, w)>j$ we have $w \omega_{j}=\omega_{j}$. Hence only the part to the left of $w$ matters in computing $u \omega_{j}$. The formulas follow from straightforward computations.

The consequences of Lemma 6.2 are that, if $h(i d, u)=h\left(i d, u^{\prime}\right)=j$, then:

(1) $h\left(\omega_{j}-u \omega_{j}\right)=j$.

(2) $\omega_{j}-u \omega_{j}$ is a root, except in type $B$ when it can also be twice a root.

(3) $\omega_{j}-u \omega_{j}$ and $\omega_{j}-u^{\prime} \omega_{j}$ are either equal or independent.

Therefore each factor

$$
u_{k-1} \omega_{i_{k}}-v \omega_{i_{k}}=u_{k-1}\left(\omega_{i_{k}}-u_{k-1}^{-1} v \omega_{i_{k}}\right)
$$

in the denominator of (5.2) is, in types $A$ and $C$, a root, and in type $B$, either a root or twice a root. Using Lemma 4.2 we conclude that each such factor cancels (over $\mathbb{Z}_{>0}$ in types $A$ and $C$, and over $\frac{1}{2} \mathbb{Z}_{>0}$ in type $B$ ) one of the factors in $\Lambda_{v}^{-}$.

To finish the proof of Theorem 6.1, we need to show that the factors in the denominator of (5.2) are pairwise independent. Suppose that is not true, and that for some chain $\gamma \in \mathcal{C}_{0}(u, v)$ and some $k<t$, the vectors $u_{k-1} \omega_{i_{k}}-v \omega_{i_{k}}$ and $u_{t-1} \omega_{i_{t}}-v \omega_{i_{t}}$ are dependent. Then $\omega_{i_{k}}-v^{-1} u_{k-1} \omega_{i_{k}}$ and $\omega_{i_{t}}-v^{-1} u_{t-1} \omega_{i_{t}}$ are also dependent. Using Lemma 6.2 we get that

$$
i_{k}=h\left(\omega_{i_{k}}-v^{-1} u_{k-1} \omega_{i_{k}}\right)=h\left(\omega_{i_{t}}-v^{-1} u_{t-1} \omega_{i_{t}}\right)=i_{t},
$$

hence $i_{k}=i_{t}$. Let $j=i_{k}=i_{t}$ be the common value. Since $\omega_{j}-v^{-1} u_{k-1} \omega_{j}$ and $\omega_{j}-v^{-1} u_{t-1} \omega_{j}$ are dependent, Lemma 6.2 implies that they must be equal, and therefore $u_{k-1} \omega_{j}-u_{t-1} \omega_{j}=0$. Then the proof of Lemma 4.2 implies that $\left\langle\omega_{j}, \beta_{k}\right\rangle=0$, and that is a contradiction, since $j=i_{k}=h\left(\beta_{k}\right)$. Therefore the factors in the denominator of (5.2) are independent, and that completes the proof of the theorem. 
Remark 6.1. If $u=s_{2}$ and $v=s_{1} s_{2} s_{1}$ in $B_{2}$, then the chain

$$
\gamma: s_{2} \stackrel{* s_{\alpha_{1}+2 \alpha_{2}}}{\longrightarrow} s_{1} s_{2} \stackrel{* s_{\alpha_{1}+\alpha_{2}}}{\longrightarrow} s_{1} s_{2} s_{1}
$$

is in $\mathcal{C}_{0}(u, v)$, but

$$
E(\gamma)=\alpha_{1}\left(\alpha_{1}+\alpha_{2}\right)\left(\alpha_{1}+2 \alpha_{2}\right) \frac{1}{2\left(\alpha_{1}+\alpha_{2}\right)} \frac{1}{\alpha_{1}+2 \alpha_{2}}=\frac{1}{2} \alpha_{1},
$$

hence in type $B$ the formula (6.4) is not necessarily integral, and the denominators can be higher powers of 2 .

A key consequence of Lemma 6.2 is that if $h(i d, u)=j$, then $\omega_{j}-u \omega_{j}$ is a root (in types $A$ and $C$ ) or a multiple of a root (it type $B$, and for $G_{2}$ ). However, in type $D$ or for $F_{4}, \omega_{j}-u \omega_{j}$ can be a sum of positive roots that is not a multiple of a root, and therefore chain contributions (5.2) are positive but not necessarily polynomial. Sabatini and Tolman [ST] developed a general procedure of grouping several contributions in order to get a positive integral formula in types $B$ and $D$.

\section{Chain formula in type A}

We apply Theorem 6.1 in the type $A$ case, for $M=\mathrm{SL}_{n}(\mathbb{C}) / B$.

If $h(\beta)=k$, then $\beta=\alpha_{k j}$ for some $j \geqslant k$ and $\left\langle\omega_{k}, \beta\right\rangle=1$. Hence all the factors in the numerator of (5.2) are equal to 1 . The denominators can be computed using the following lemma.

Lemma 7.1. Let $u \in S_{n}$. If $h(i d, u)=j$, then

$$
\omega_{j}-u \omega_{j}=x_{j}-x_{u(j)} .
$$

Proof. Let $u=s_{k} s_{k-1} \cdots s_{j} w$ be the decomposition (6.5). Then

$$
\omega_{j}-u \omega_{j}=\alpha_{j}+\cdots \alpha_{k}=x_{j}-x_{k+1},
$$

and since $h(i d, w)>j$, we have $k+1=u(j)$.

If $\gamma$ is the saturated chain (6.2) as in Theorem 6.1, then

$$
u_{k-1} \omega_{i_{k}}-v \omega_{i_{k}}=u_{k-1}\left(\omega_{i_{k}}-u_{k-1}^{-1} v \omega_{i_{k}}\right)=x_{u_{k-1}\left(i_{k}\right)}-x_{v\left(i_{k}\right)},
$$

and therefore

$$
E(\gamma)=\left[\prod_{(a, b) \in \operatorname{Inv}(v)}\left(x_{a}-x_{b}\right)\right] \prod_{k=1}^{m} \frac{1}{x_{u_{k-1}\left(i_{k}\right)}-x_{v\left(i_{k}\right)}} .
$$

We summarize the results of this section and formulate our version of a type $A$ positive formula for $\tau_{u}(v)$. Let $C_{0}(u, v)$ be the set of saturated chains

$$
\gamma: u=u_{0} \stackrel{*\left(i_{1}, j_{1}\right)}{\longrightarrow} u_{1} \stackrel{*\left(i_{2}, j_{2}\right)}{\longrightarrow} u_{2} \rightarrow \cdots \rightarrow u_{m-1} \stackrel{*\left(i_{m}, j_{m}\right)}{\longrightarrow} u_{m}=v
$$


that satisfy the condition

$$
i_{1} \leqslant i_{2} \leqslant \cdots \leqslant i_{m}
$$

where $*(i, j)$ means that we are multiplying to the right by the transposition that swaps $i$ and $j$. For such a chain $\gamma$, let

$$
\operatorname{Inv}(v, \gamma)=\operatorname{Inv}(v) \backslash\left\{\left(u_{k-1}\left(i_{k}\right), v\left(i_{k}\right)\right) \mid k=1, \ldots, m\right\}
$$

and

$$
E(\gamma)=\prod_{(a, b) \in \operatorname{Inv}(v, \gamma)}\left(x_{a}-x_{b}\right)
$$

Theorem 7.1. The restriction of $\tau_{u}$ at $v$ is given by

$$
\tau_{u}(v)=\sum_{\gamma \in C_{0}(u, v)} E(\gamma)=\sum_{\gamma \in C_{0}(u, v)} \prod_{(a, b) \in \operatorname{Inv}(v, \gamma)}\left(x_{a}-x_{b}\right) .
$$

Example 7.1. Consider the permutations $u=2143$ and $v=3421$ in $S_{4}$. There are two chains in $\mathcal{C}_{0}(2143,3421)$ :

$$
\begin{array}{ll}
\gamma_{1}: & 2143 \stackrel{*(1,4)}{\longrightarrow} 3142 \stackrel{*(2,3)}{\longrightarrow} 3412 \stackrel{*(3,4)}{\longrightarrow} 3421, \\
\gamma_{2}: & 2143 \stackrel{*(1,4)}{\longrightarrow} 3142 \stackrel{*(2,4)}{\longrightarrow} 3241 \stackrel{*(2,3)}{\longrightarrow} 3421 .
\end{array}
$$

For the first chain $u_{0}=2143, u_{1}=3142, u_{2}=3412$, and $u_{3}=v=3421$. The edge $2143 \stackrel{*(1,4)}{\longrightarrow} 3142$ deletes the factor $x_{u_{0}(1)}-x_{v(1)}=x_{2}-x_{3}$. The edge $3142 \stackrel{*(2,3)}{\longrightarrow} 3412$ deletes the factor $x_{u_{1}(2)}-x_{v(2)}=x_{1}-x_{4}$. The edge $3412 \stackrel{*(3,4)}{\longrightarrow} 3421$ deletes the factor $x_{u_{2}(3)}-x_{v(3)}=x_{1}-x_{2}$. Therefore,

$$
\begin{aligned}
E\left(\gamma_{1}\right) & =\frac{\left(x_{2}-x_{3}\right)\left(x_{1}-x_{3}\right)\left(x_{2}-x_{4}\right)\left(x_{1}-x_{4}\right)\left(x_{1}-x_{2}\right)}{\left(x_{2}-x_{3}\right)\left(x_{1}-x_{4}\right)\left(x_{1}-x_{2}\right)} \\
& =\left(x_{1}-x_{3}\right)\left(x_{2}-x_{4}\right)=\left(\alpha_{1}+\alpha_{2}\right)\left(\alpha_{2}+\alpha_{3}\right) .
\end{aligned}
$$

Similarly,

$$
\begin{aligned}
E\left(\gamma_{2}\right) & =\frac{\left(x_{2}-x_{3}\right)\left(x_{1}-x_{3}\right)\left(x_{2}-x_{4}\right)\left(x_{1}-x_{4}\right)\left(x_{1}-x_{2}\right)}{\left(x_{2}-x_{3}\right)\left(x_{1}-x_{4}\right)\left(x_{2}-x_{4}\right)} \\
& =\left(x_{1}-x_{3}\right)\left(x_{1}-x_{2}\right)=\left(\alpha_{1}+\alpha_{2}\right) \alpha_{1} .
\end{aligned}
$$

Hence

$$
\tau_{2143}(3421)=E\left(\gamma_{1}\right)+E\left(\gamma_{2}\right)=\left(\alpha_{1}+\alpha_{2}\right)\left(\alpha_{1}+\alpha_{2}+\alpha_{3}\right) .
$$




\section{Chains and subwords}

In this section we give a general construction connecting ascending chains and subwords, valid for all Weyl groups and orderings of simple roots.

Let $u \prec v$ and let $I=\left[i_{1}, \ldots, i_{k}\right]$ be a reduced word for $v$. Let $\mathcal{A}(u, v)$ be the set of all ascending chains

$$
\gamma: u=u_{0} \stackrel{* s_{\beta_{1}}}{\longrightarrow} u_{1} \stackrel{* s_{\beta_{2}}}{\longrightarrow} u_{2} \rightarrow \cdots \rightarrow u_{m-1} \stackrel{* s_{\beta_{m}}}{\longrightarrow} u_{m}=v,
$$

from $u$ to $v$, not necessarily of maximal length, and let $\mathcal{C}(u, v)$ be the subset of $\mathcal{A}(u, v)$ consisting of the chains that satisfy $h\left(\beta_{1}\right) \leqslant \cdots \leqslant h\left(\beta_{m}\right)$. Then

$$
\mathcal{C}(u, v) \cap \Sigma(u, v)=\mathcal{C}_{0}(u, v) \subset \mathcal{C}(u, v) \subset \mathcal{A}(u, v) .
$$

Let $\mathcal{S}(u, I)$ be the set of subwords of $I$ that generate words for $u$ by deleting the zeroes; then $\mathcal{R}(u, I)$ is a subset of $\mathcal{S}(u, v)$. We define a function $F_{I}: \mathcal{A}(u, v) \rightarrow \mathcal{S}(u, I)$ as follows. Let $\gamma$ be the ascending chain (8.1). We use the edges of $\gamma$, in reverse order, to delete letters from $I$ and get a subword of $I$ that is a word for $u$. Since $u_{m-1}=v s_{\beta_{m}} \prec v$, the Strong Exchange Property implies that there exists a $j$ (unique, since $I$ is reduced) such that

$$
u_{m-1}=s_{i_{1}} \cdots s_{i_{j-1}} s_{i_{j+1}} \cdots s_{i_{k}},
$$

hence $I_{m-1}=\left[i_{1}, \ldots, i_{j-1}, 0, i_{j+1}, \ldots, i_{k}\right] \in \mathcal{S}\left(u_{m-1}, I\right)$ is a subword of $I$ that is a word for $u_{m-1}$. Similarly, $u_{m-2}=u_{m-1} s_{\beta_{m-1}}$ and $\ell\left(u_{m-2}\right)<\ell\left(u_{m-1}\right)$, so there exists a subword of $I_{m-1}$, obtained by deleting exactly one letter from $I_{m-1}$, that is a word for $u_{m-2}$. However, since $\ell\left(u_{m-1}\right)$ is not necessarily equal to $\ell(v)-1$, the word $I_{m-1}$ may be non-reduced, and in this case the uniqueness of the deleted letter is not guaranteed. We choose the subword for which the deleted letter is the rightmost choice, and get a subword $I_{m-2}$ of $I_{m-1}$ (hence of $I$ ), that is a word, not necessarily reduced, for $u_{m-2}$. Continuing this process, with the same rule for making a choice, if needed, we get a sequence $I_{m-1}, \ldots, I_{0}$ of subwords of $I$ such that $I_{k} \in \mathcal{S}\left(u_{k}, I\right)$ and $I_{k}$ is obtained from $I_{k+1}$ by deleting one letter. We define $F_{I}(\gamma)=I_{0}$, the last subword in the sequence.

Example 8.1. Let $u=s_{1}$ and $v=s_{1} s_{2} s_{1} s_{2}$ in the Weyl group of the root system $C_{2}$. Let $I=[1,2,1,2]$ be a reduced word for $v$ and let

$$
\gamma: \quad s_{1} \stackrel{* s_{\alpha_{1}+\alpha_{2}}}{\longrightarrow} s_{2} s_{1} \stackrel{* s_{\alpha_{1}+2 \alpha_{2}}}{\longrightarrow} s_{1} s_{2} s_{1} \stackrel{* s_{\alpha_{2}}}{\longrightarrow} s_{1} s_{2} s_{1} s_{2}
$$

be an ascending chain of maximal length from $u$ to $v$. Since $s_{\alpha_{1}+\alpha_{2}}=s_{1} s_{2} s_{1}$ and $s_{\alpha_{1}+2 \alpha_{2}}=s_{2} s_{1} s_{2}$, the subword $F_{I}(\gamma)$ is computed as follows:

$$
\begin{aligned}
& {[1,2,1,2] \cdot[2]=[1,2,1,0]=I_{2},} \\
& {[1,2,1,0] \cdot[2,1,2]=[0,2,1,0]=I_{1},} \\
& {[0,2,1,0] \cdot[1,2,1]=[0,0,1,0]=I_{0},}
\end{aligned}
$$

hence $F_{I}(\gamma)=[0,0,1,0]$. 
Note that if $\gamma \in \Sigma(u, v)$, then $\ell\left(u_{k-1}\right)=\ell\left(u_{k}\right)-1$ for all $k$, hence the words $I_{m-1}, \ldots, I_{1}, I_{0}$ are reduced. Therefore at each stage there is only one possibility for the deleted letter and no choice is necessary.

Lemma 8.1. Let $\gamma \in \mathcal{A}(u, v)$ be an ascending chain. Then

$$
F_{I}(\gamma) \in \mathcal{R}(u, I) \Longleftrightarrow \gamma \in \Sigma(u, v) .
$$

Moreover, if $F_{I}: \mathcal{C}(u, v) \rightarrow \mathcal{S}(u, I)$ is bijective, then $F_{I}: \mathcal{C}_{0}(u, v) \rightarrow \mathcal{R}(u, I)$ is also bijective.

Proof. Let $\gamma \in \mathcal{A}(u, v)$. Then $F_{I}(\gamma) \in \mathcal{R}(u, I)$ if and only if $F_{I}(\gamma)$ has $\ell(u)$ non-zero letters. This happens if and only if we delete $\ell(v)-\ell(u)$ letters from $I$, and since every deleted letter corresponds to an edge of $\gamma$, if and only if $\gamma$ has $\ell(v)-\ell(u)$ edges. In other words, if and only if $\gamma$ is an ascending chain of maximal length. If $\gamma \in \mathcal{C}_{0}(u, v)$ then $\gamma \in \Sigma(u, v)$, hence $F_{I}(\gamma) \in \mathcal{R}(u, I)$. Therefore $F_{I}: \mathcal{C}_{0}(u, v) \rightarrow \mathcal{R}(u, I)$ is well-defined.

Suppose that $F_{I}: \mathcal{C}(u, v) \rightarrow \mathcal{S}(u, I)$ is bijective. Then the restriction of $F_{I}$ to $\mathcal{C}_{0}(u, v)$ is injective. Moreover, if $J \in \mathcal{R}(u, I)$, then there exists $\gamma \in \mathcal{C}(u, v)$ such that $F_{I}(\gamma)=J$. But then $\gamma \in \mathcal{C}(u, v) \cap \Sigma(u, v)=\mathcal{C}_{0}(u, v)$, hence $F_{I}: \mathcal{C}_{0}(u, v) \rightarrow \mathcal{R}(u, I)$ is also surjective.

\section{Equivalence of positive formulas}

We show that in the case $G=\mathrm{SL}_{n}(\mathbb{C})$, the chain formula (7.1) is an alternative formulation of the subword formula (3.1), by proving that, for a particular choice of a reduced word $I$ for $v$, the function $F_{I}$ restricts to a bijection $F_{I}: \mathcal{C}_{0}(u, v) \rightarrow \mathcal{R}(u, I)$ and $\operatorname{SC}\left(F_{I}(\gamma), I\right)=E(\gamma)$ for all $\gamma \in \mathcal{C}_{0}(u, v)$. Let $I(v)$ be the reduced word for $v$ constructed inductively using (6.5). Then

$$
I(v)=\left[I_{1}, I_{2}, \ldots, I_{n-1}\right],
$$

such that, for all $j=1, \ldots, n-1$,

- $I_{j}=\left[k_{j}, k_{j}-1, \ldots, j+1, j\right]$ or $I_{j}=[]$, and

- $h\left(s_{I_{1}} \cdots s_{I_{j}}, v\right)=h\left(i d, s_{I_{j}} \cdots s_{I_{1}} v\right)=h\left(i d, s_{I_{j+1}} \cdots s_{I_{n-1}}\right)>j$.

Example 9.1. If $v=s_{2} s_{1} s_{3} s_{2} s_{3}$, then $I_{1}=[2,1], I_{2}=[3,2]$, and $I_{3}=[3]$.

We can now prove the equivalence of the two positive formulas in type $A$.

Theorem 9.1. Let $u, v \in S_{n}$ and let $I=I(v)$. Then $F_{I}: \mathcal{C}_{0}(u, v) \rightarrow \mathcal{R}(u, I)$ is a bijection and

$$
\mathrm{SC}\left(F_{I}(\gamma), I\right)=E(\gamma)
$$

for all chains $\gamma \in \mathcal{C}_{0}(u, v)$.

Proof. In $[\mathbf{Z}]$ we proved that $F_{I}: \mathcal{C}(u, v) \rightarrow \mathcal{S}(u, I)$ is a bijection. Then, by Lemma 8.1, the map $F_{I}: \mathcal{C}_{0}(u, v) \rightarrow \mathcal{R}(u, I)$ is also a bijection. 
To prove that $\operatorname{SC}\left(F_{I}(\gamma), I\right)=E(\gamma)$, we show that the factor of $\Lambda_{v}^{-}$cancelled by an edge of $\gamma$ is the same as the factor cancelled by the corresponding deleted letter from $I$. In $[\mathbf{Z}]$ we also proved that if

$$
\gamma: u=u_{0} \stackrel{* s_{\beta_{1}}}{\longrightarrow} u_{1} \stackrel{* s_{\beta_{2}}}{\longrightarrow} u_{2} \rightarrow \cdots \rightarrow u_{m-1} \stackrel{* s_{\beta_{m}}}{\longrightarrow} u_{m}=v
$$

is a chain in $\mathcal{C}_{0}(u, v)$, then,

(1) the edge $u_{k-1} \stackrel{* s_{\beta_{k}}}{\longrightarrow} u_{k}$ deletes a letter from $I_{h\left(\beta_{k}\right)}$, and

(2) the letters deleted in $I_{j}$ are deleted from left to right.

Let $u_{k-1} \rightarrow u_{k}=u_{k-1} s_{\beta_{k}}$ be an edge of $\gamma$ and let $j=h\left(\beta_{k}\right)$.

Suppose that $h\left(\beta_{k}\right)=\cdots=h\left(\beta_{k+q-1}\right)=j<h\left(\beta_{k+q}\right)$, hence $\beta_{k}$ is the $q$ th occurrence of $j$ from right to left. Let

$$
J_{k}=\left[I_{1}, \ldots, I_{j-1}, I_{j}^{\prime}, I_{j+1}^{\prime}, \ldots, I_{n-1}^{\prime}\right] \in \mathcal{R}\left(u_{k}, I(v)\right)
$$

be the subword obtained after using $s_{\beta_{m}}, \ldots, s_{\beta_{k+1}}$ to delete letters from $I(v)$, and let

$$
J_{k-1}=\left[I_{1}, \ldots, I_{j-1}, I_{j}^{\prime \prime}, I_{j+1}^{\prime}, \ldots, I_{n-1}^{\prime}\right] \in \mathcal{R}\left(u_{k-1}, I(v)\right)
$$

be the subword obtained after using $s_{\beta_{k}}$ to delete one more letter from $J_{k}$. Then $I_{j}^{\prime}$ is of the form

$$
I_{j}^{\prime}=\left[k_{j}, \ldots, \widehat{j_{1}}, \ldots, \widehat{j_{q-1}}, \ldots, j\right] \subset I_{j},
$$

and

$$
I_{j}^{\prime \prime}=\left[k_{j}, \ldots, \widehat{j_{1}}, \ldots, \widehat{j_{q-1}}, \ldots, \widehat{j_{q}}, \ldots, j\right] \subset I_{j}^{\prime} \subset I_{j},
$$

with $j_{1}>j_{2}>\cdots>j_{q}$, since the letters in $I_{j}$ are deleted from left to right.

The factor of $\Lambda_{v}^{-}$cancelled by the edge $u_{k-1} \rightarrow u_{k}$ is

$$
\begin{aligned}
u_{k-1} \omega_{j}-v \omega_{j} & =\left(u_{k-1} \omega_{j}-u_{k} \omega_{j}\right)+\cdots+\left(u_{k+q-2} \omega_{j}-u_{k+q-1} \omega_{j}\right) \\
& =\left\langle\omega_{j}, \beta_{k}\right\rangle u_{k-1} \beta_{k}+\cdots+\left\langle\omega_{j}, \beta_{k+q-1}\right\rangle u_{k+q-2} \beta_{k+q-1} \\
& =s_{I_{1}} \cdots s_{I_{j-1}} s_{k_{j}} \cdots s_{j_{1}+1}\left(\widehat{s_{j_{1}}} \cdots \widehat{s_{j_{2}}} \cdots \widehat{s_{j_{q}}} \alpha_{j_{q}}+\cdots+\alpha_{j_{1}}\right) .
\end{aligned}
$$

But if $j_{1}>j_{2}$, then

$$
\widehat{s_{j_{1}}} \cdots \widehat{s_{j_{2}}} \alpha_{j_{2}}+\alpha_{j_{1}}=s_{j_{1}} \cdots \widehat{s_{j_{2}}} \alpha_{j_{2}}
$$

and then, by induction on $q$,

$$
\widehat{s_{j_{1}}} \cdots \widehat{s_{j_{2}}} \cdots \widehat{s_{j_{q}}} \alpha_{j_{q}}+\cdots+\alpha_{j_{1}}=s_{j_{1}} \cdots s_{j_{q-1}} \cdots \widehat{s_{j_{q}}} \alpha_{j_{q}} \text {. }
$$

Therefore,

$$
u_{k-1} \omega_{j}-v \omega_{j}=s_{I_{1}} \cdots s_{I_{j-1}} s_{k_{j}} \cdots s_{j_{q}+1} \alpha_{j_{q}}
$$

and that is precisely the factor of $\Lambda_{v}^{-}$cancelled by the missing letter $j_{q}$, letter that has been deleted by the edge $u_{k-1} \rightarrow u_{k}$. 
In type $C$ the two formulas are different, as shown by the following example. Let $u=s_{1}$ and $v=s_{1} s_{2} s_{1} s_{2}$ in $C_{2}$. Then $I_{1}=[1,2,1,2]$ and $I_{2}=[2,1,2,1]$ are the only reduced words for $v$, and each of them has two subwords that are reduced words for $u$. Hence whether $I=I_{1}$ or $I=I_{2}$, there are two subwords in $\mathcal{R}(u, I)$. There is only one saturated chain from $u$ to $v$ satisfying (6.3),

$$
\gamma: \quad s_{1} \stackrel{* s_{\alpha_{1}+\alpha_{2}}}{\longrightarrow} s_{2} s_{1} \stackrel{* s_{\alpha_{1}+2 \alpha_{2}}}{\longrightarrow} s_{1} s_{2} s_{1} \stackrel{* s_{\alpha_{2}}}{\longrightarrow} s_{1} s_{2} s_{1} s_{2}
$$

hence there is only one chain in $\mathcal{C}_{0}(u, v)$. The subword formula has two terms in the sum, but the chain formula has only one term, so the two formulas are different. (In general, however, the number of chains in $\mathcal{C}_{0}(u, v)$ is not necessarily smaller than the number of subwords in $\mathcal{R}(u, I)$.)

\section{References}

[AJS] H. H. Andersen, J. C. Jantzen and W. Soergel, Representations of quantum groups at a pth root of unity and of semisimple groups in characteristic $p$ : independence of $p$, Astérisque 220 (1994), 297-302.

[BB] A. Björner and F. Brenti, Combinatorics of Coxeter groups, Graduate Texts in Mathematics, 231. Springer, New York, 2005.

[B] S. Billey, Kostant polynomials and the cohomology ring for $G / B$, Duke Math. J. 96 (1) (1999), 205-224.

[GHZ] V. Guillemin, T. Holm and C. Zara, A GKM description of the equivariant cohomology ring of a homogeneous space, J. Algebraic Combin. 23 (1) (2006), 2141.

[GT] R. Goldin and S. Tolman, Towards generalizing Schubert calculus in the symplectic category. J. Sympl. Geom. 7 (4) (2009), 449-473.

[K] A. Knutson, A Littelmann-type formula for Duistermaat-Heckman measures, Invent. Math. 135 (1) (1999), 185-200.

[KK] B. Kostant and S. Kumar, The nil Hecke ring and cohomology of G/P for a KacMoody group G, Adv. Math. 62 (1986) 187-237.

[KM] A. Knutson and E. Miller, Gröbner geometry of Schubert polynomials, Ann. Math. 161 (3) (2005), 1245-1318.

[ST] S. Sabatini and S. Tolman, A simpler path formula for equivariant cohomology. Preprint.

[Z] C. Zara, Chains, subwords, and fillings: strong equivalence of three definitions of the Bruhat order, Electron. J. Combin. 13 (1) (2006), N5.

Department of Mathematics

University of MASSACHUSETtS AT Boston

100 MORRISSEY BLVD.

Boston, MA 02125

E-mail address: czara@math.umb.edu

Received 9/29/2008, accepted 2/24/2010 
I would like to thank Rebecca Goldin, Sue Tolman, Silvia Sabatini, and Allen Knutson for explaining their work, and to all of the above and Victor Guillemin for many stimulating discussions and valuable comments while writing this paper. Many thanks are also due to John Stembridge for writing the Maple packages coxeter and weyl, which have been used to verify several conjectured formulas before proving them, and to a meticulous referee whose comments and suggestions improved the presentation of this paper. 
